$\mathbb{T}$ periodica polytechnica

\author{
Electrical Engineering \\ and Computer Science

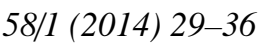 \\ doi: $10.3311 /$ PPee.7030
}

Creative Commons Attribution (i)

RESEARCH ARTICLE

\section{Model-based Angiogenic Inhibition of Tumor Growth using Adaptive Fuzzy Techniques}

Annamária Szeles / Dániel András Drexler / Johanna Sápi / István Harmati / Levente

Kovács

Received 2013-09-02, accepted 2013-11-13

\begin{abstract}
Fighting tumors is one of the most important problems of medical research. In this paper, antiangiogenic cancer therapy is investigated through its mathematical model. This tumor treatment method targets the endothelium of a growing tumor and belongs to the targeted molecular therapies. The aim of the therapy is not to eliminate the entire tumor, but to decrease the tumor to a minimal volume. An advantage of applying antiangiogenic treatment is that tumor cells show lower tendency of becoming resistant to the applied drugs. Adaptive fuzzy control is implemented for a simplified model to elaborate a control technique which is able to handle the effects of parameter perturbations and uncertainties while keeping the daily and total inhibitor inlet under a given limit.
\end{abstract}

\section{Keywords}

biomedical systems $\cdot$ tumor treatment $\cdot$ soft computing $\cdot$ fuzzy systems $\cdot$ adaptive control

\section{Annamária Szeles}

Department of Control Engineering and Information Technology, Budapest University of Technology and Economics,

Magyar tudósok krt. 2., H-1117 Budapest, Hungary

e-mail: szeles.annam@gmail.com

\section{Dániel András Drexler}

Department of Control Engineering and Information Technology, Budapest University of Technology and Economics,

Magyar tudósok krt. 2., H-1117 Budapest, Hungary

e-mail: drexler@iit.bme.hu

\section{Johanna Sápi}

John von Neumann Faculty of Informatics, Óbuda University,

Bécsi út 96/B, H-1034 Budapest, Hungary

e-mail: sapi.johanna@phd.uni-obuda.hu

\section{István Harmati}

Department of Control Engineering and Information Technology, Budapest University of Technology and Economics,

Magyar tudósok krt. 2., H-1117 Budapest, Hungary

e-mail: harmati@iit.bme.hu

\section{Levente Kovács}

John von Neumann Faculty of Informatics, Óbuda University, Bécsi út 96/B, H-1034 Budapest, Hungary

e-mail: kovacs.levente@nik.uni-obuda.hu

\section{Introduction}

Cancer treatment is one of the most rapidly developing research fields of medicine. Modern techniques including targeted molecular therapies target only cancerous cells opposed to conventional therapies, e.g. chemotherapy or radiotherapy where both tumorous and normal cells are affected by the applied drugs. In this paper, antiangiogenic therapy is investigated, this method inhibits the tumor from growing own blood vessel cappilaries which serve the tumor to nourish from the host body [1, 2]. The most important advantage of the therapy is that patients do not have to suffer from severe side-effects during antiangiogenic treatment, and tumor cells do not develop intrinsic resistance to the angiogenic inhibitors.

Philip Hahnfeldt et al. proposed a biologically validated, population based model described by ordinary differential equations in 1999 [3]. Since then, the mathematical model was modified and reformulated many times [4,5]. In this paper a simplified second order model is used to investigate adaptive fuzzy control methodology. For the same model, bang-singular-bang control was designed in [6] and optimal linear control was implemented in [7]. A set-valued protocol was elaborated in [8].

More different approaches were used to handle the nonlinearity of the system. Working point linearization was performed to design and analyze state-space and robust control in former papers [7, 9, 10], flatness based technique was proposed in [11] for the original model using constant Hurwitz polinomial, and in [12] for the simplified model using tumor volume dependent Hurwitz polinomials. Linear controllers could not handle the nonlinearity of the system appropriately, since the magnitude of the control input was infeasible, and the avascular state of the tumor was not attained. Flatness based techniques were not efficient when nominal model parameters changed due to parametric uncertainties. In this paper, adaptive fuzzy control is designed, that can handle both the nonlinearity of the system and the effects of model parameter perturbations.

Section 2 focuses on the biomedical background of tumor growth and applied treatments. Section 3 presents the nonlinear model of tumor growth under angiogenic inhibition. In Section 4 the theoretical background of fuzzy control is shortly in- 
troduced, and Section 5 details the controller design and related simulations. The paper ends with the conclusions in Section 6 .

\section{Biomedical background}

Medical practice [13] refers to chemotherapy [14], radiotherapy [15] and surgical intervention [16] as conventional treatments. These therapies aim to prevent cell division and the rapid proliferation of tumors. However, these techniques include many non-desired side effects: affecting normal tissue and the development of intrinsic resistance (pre-existing resistance) to the applied drugs in the case of chemotherapy, the damage of healthy tissue DNA in the case of radiotherapy, and the injury of nearby normal tissue in the case of curative surgary. Resistance to the drugs applied during chemotherapy already exists before the treatment in approximately $50 \%$ of cancer cases [17].

An in vivo tumor, after passing the initial avascular state at a volume of 1-2 $\mathrm{mm}^{3}$, starts to recruit own blood vessels in order to take up nutritiants and oxygen. This phenomenon, called tumor-induced angiogenesis [2], is captured and inhibited by the antiangiogenic therapy [1, 18]. Antiangiogenic drugs are able to block and disrupt the vascular endothelial growth of the tumor, thus tumor regresses until reaching an avascular state which can be maintained using nontoxic inhibitor concentrations [19,20]. Another advantage of antiangiogenic treatments is that these evoke only aquired resistance (resistance induced by the drug) instead of intrinsic resistance. New results of medical research give alternative solutions to overcome drug induced resistance [21], which makes these cancer treatment methods more prosperous.

Prevalent angiogenic inhibitors are endostatin [22] and bevacizumab [23]. The model formulation presented in this paper is based on therapies which used endostatin.

\section{Dynamical model of tumor growth}

The mathematical formalism describing tumor growth dynamics under angiogenic signaling was posed by Philip Hanhfeldt et al. [3]. The model was biologically validated through animal experiments in which mice were injected with carcinoma cells. The differential equations which describe the model are the following:

$$
\begin{aligned}
& \dot{x}_{1}=-\lambda_{1} x_{1} \ln \left(\frac{x_{1}}{x_{2}}\right) \\
& \dot{x}_{2}=\tilde{b} x_{1}-\tilde{d} x_{1}^{\frac{2}{3}} x_{2}-\tilde{e} x_{2} x_{3} \\
& \dot{x}_{3}=\int_{0}^{t} u\left(t^{\prime}\right) \exp \left(-\lambda_{3}\left(t-t^{\prime}\right)\right) d t^{\prime}
\end{aligned}
$$

where $x_{1}$ is the tumor volume $\left(\mathrm{mm}^{3}\right), x_{2}$ is the supporting vasculature volume $\left(\mathrm{mm}^{3}\right)$ which refers to the volume of the endothelium, $x_{3}$ is the inhibitor serum level $(\mathrm{mg} / \mathrm{kg})$, and $u$ is the inhibitor administration rate $(\mathrm{mg} / \mathrm{kg} /$ day $)$. The parameters characteristic for the animals and the Lewis lung carcinoma are: $\lambda_{1}=$ $0.192\left(\right.$ day $\left.^{-1}\right), \tilde{b}=5.85\left(\right.$ day $\left.^{-1}\right), \tilde{d}=0.00873\left(\right.$ day $\left.^{-1} \mathrm{~mm}^{-2}\right)$, the

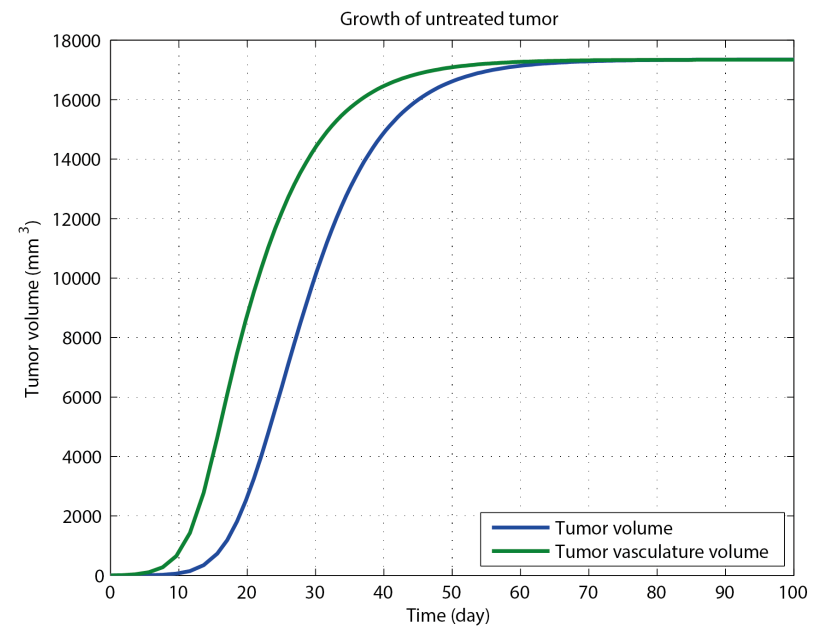

Fig. 1. Growth of untreated tumor

parameters characteristic for the applied inhibitor (endostatin) are: $\tilde{e}=0.66\left(\mathrm{day}^{-1}(\mathrm{mg} / \mathrm{kg})^{-1}\right), \lambda_{3}=1.3\left(\mathrm{day}^{-1}\right)$. The nonlinear model involves the following phenomenologies: tumor growth slowdown, as tumor cells tax all available support; intrinsic stimulation and inhibition originating from the tumor cells; inhibition due to the applied drug; and the clearance of the inhibitor. The latter effect is not described by the simplified model, since zero-order pharmacokinetics is used instead of first-order pharmacokinetics. The second order system chosen for controller design and simulations is the following:

$$
\begin{aligned}
\dot{x}_{1} & =-\lambda x_{1} \ln \left(\frac{x_{1}}{x_{2}}\right) \\
\dot{x}_{2} & =\tilde{b} x_{1}-\tilde{d} x_{1}^{\frac{2}{3}} x_{2}-\tilde{e} x_{2} u \\
y & =x_{1}
\end{aligned}
$$

where $x_{1}$ is the tumor volume $\left(\mathrm{mm}^{3}\right), x_{2}$ is the vasculature volume $\left(\mathrm{mm}^{3}\right)$, and $u$ is the serum level of the administered inhibitor $(\mathrm{mg} / \mathrm{kg})$. The model parameter $\lambda$ equals to $\lambda_{1}$ in (1)-(3). The measured output of the system is the tumor volume (6). The nonlinear behavior of the system without angiogenic inhibition is presented in Fig. 1 .

\section{Adaptive fuzzy design}

The elaboration of fuzzy control was inspired by human logic and decision-making. Instead of calculating the control input based on crisp values, the algorithm uses fuzzy sets [24], which model both the uncertainty of the measured output value (measurement noises, disturbances etc.) and the uncertainty of decision-making in certain parts of the control input domain. The steps of the algorithm are the following [25]:

\section{Normalization}

\section{Fuzzyfication of the crisp input}

3 Calculation of the controller output based on fuzzy rules and the input and the output fuzzy sets 
4 Defuzzyfication of the fuzzy output

\section{Denormalization}

In our approach, rules are defined in the natural domain of the input (tumor volume) and the output (serum level), thus normalization and denormalization can be eliminated, however domain limits have to be considered.

In soft computing theory, there are different types of adaptive fuzzy control. According to control characteristics, indirect or direct control, and according to parameter tuning method, $1^{\text {st }}$ type and $2^{\text {nd }}$ type control can be distinguished [26]:

- Indirect: simultaneously model identification is performed, and the corresponding control input is determined.

- Direct: control input is calculated without performing model identification.

- $1^{\text {st }}$ type: only output membership function parameters are tuned.

- $2^{\text {nd }}$ type: both input and output membership function parameters are tuned.

In this study, $1^{\text {st }}$ type direct adaptive fuzzy control is presented. Direct method is chosen instead of indirect one, since estimation can be given to the model related control parameters. $1^{\text {st }}$ type was chosen instead of $2^{\text {nd }}$ type, since input membership functions can be posed based on a priori information without parameter tuning.

The expected differential equation of the system is in the form of

$$
y^{(n)}=f(x)+g(x) u
$$

under the constraint that lower order derivatives of $y$ do not depend on $u$ explicitly. The constraint is fulfilled, related calculations were published in [12]. The notations are the following: $n$ is the differential order of the system $(n=2), f(x) \in \mathbb{R}, g(x) \in \mathbb{R}$ (since the controlled system is a single-input single-output system), and $g(x)>0$. The latter condition is not valid for the tumor growth model, since $g(x)=-\tilde{e} x_{2}$ where $\tilde{e}>0$ and $x_{2} \geq 0$. Thus, the following concept has to be used in adaptive control design:

$$
y^{(n)}=f(x)+|g(x)|(-u)
$$

which means that the control input is calculated according to the equations of direct adaptive fuzzy control, however the additive inverse of the calculated control input is sent to the nonlinear system.

The reference signal and its derivatives are:

$$
\begin{aligned}
& y_{\text {ref }}(t)=\left(x_{\text {init }}-1\right) e^{\frac{-t}{T}}+1 \\
& \dot{y}_{\text {ref }}(t)=-\frac{1}{T}\left(x_{\text {init }}-1\right) e^{\frac{-t}{T}} \\
& \ddot{y}_{\text {ref }}(t)=\frac{1}{T^{2}}\left(x_{\text {init }}-1\right) e^{\frac{-t}{T}}
\end{aligned}
$$

which describes exponential decrease ( $T=1 / 0.35$ day) from the initial tumor volume $\left(x_{\text {init }}=2000 \mathrm{~mm}^{3}\right)$ to the desired plateau $\left(1 \mathrm{~mm}^{3}\right)$, since tumor volume can not be decreased to zero physiologically, tumor regresses only to a minimal volume, at which it is able to nourish from the host body without growing own vascular endothelium.

\subsection{Stability}

Error dynamics of the closed-loop system can be prescribed using the $k_{i}$ coefficients, collected in $\bar{k}=\left[\begin{array}{lll}k_{1} & \ldots & k_{n}\end{array}\right]$, and the tracking error $\left(e=y_{r e f}-y\right)$ and its $e^{(i)}$ derivatives, collected in $\bar{e}=\left[\begin{array}{lll}e^{(n-1)} & \ldots & e\end{array}\right]$. The differential order of the error dynamics is equal to the differential order of the system. The error dynamics is described by

$$
0=e^{(n)}+k_{1} e^{(n-1)}+\cdots+k_{n} e .
$$

In our case, the differential order of the error dynamics is $n=2$.

If $f(x)$ and $g(x)$ were known exactly, the control input could be determined as follows:

$$
u^{*}=g^{-1}\left(y_{r e f}^{(n)}-f+<\bar{k}, \bar{e}>\right),
$$

which secures the exponential decrease of the tracking error.

In a general case, when $f(x)$ and $g(x)$ are unknown, the control input has two components: $u_{c}$ is the controller output of the direct fuzzy controller, and $u_{s}$ is the controller output of the supervisory control, which secures the stability of the closed-loop system, hence

$$
u=u_{c}+u_{s}=u^{*}+\left(u_{c}+u_{s}-u^{*}\right),
$$

in the last term $u^{*}$ is the ideal control. The differential equation of the system and error dynamics can be calculated in a general case as follows:

$$
\begin{aligned}
y^{(n)} & =y_{\text {ref }}^{(n)}+<\bar{k}, \bar{e}>+g\left(u_{c}+u_{s}-u^{*}\right) \\
e^{(n)} & =y_{r e f}^{(n)}-y^{(n)}=-<\bar{k}, \bar{e}>-g\left(u_{c}+u_{s}-u^{*}\right) \\
\dot{\bar{e}} & =\Lambda_{c} \bar{e}+b_{c} g\left(u^{*}-u_{c}-u_{s}\right)
\end{aligned}
$$

where

$$
\Lambda_{c}=\left[\begin{array}{cccc}
0 & 1 & \ldots & 0 \\
\vdots & \vdots & \ddots & \\
0 & 0 & & 1 \\
-k_{n} & -k_{n-1} & \ldots & -k_{1}
\end{array}\right] \quad b_{c}=\left[\begin{array}{c}
0 \\
\vdots \\
0 \\
1
\end{array}\right]
$$

To guarantee the stability of the closed-loop system, Lyapunov stability has to be investigated. For the current system, the Lyapunov equation is

$$
\Lambda_{c}^{T} P+P \Lambda_{c}=-Q
$$

where $Q>0$ prescribes the transient behavior of the system, and the symmetric $P>0$ is the solution of the Lyapunov equation. Since the biomedical model is a nonlinear system, further 
analyzis is necessary: we have to find an appropriate Lyapunov function, and prove that its derivative is negative in the entire domain. The Lyapunov function is constructed from the solution of the Lyapunov equation and the tracking error:

$$
V=\frac{1}{2}<P \bar{e}, \bar{e}>
$$

whose derivative has to be calculated to investigate closed-loop stability,

$$
\begin{aligned}
\frac{\mathrm{d} V}{\mathrm{~d} t} & =\frac{1}{2}<P \dot{\bar{e}}, \bar{e}>+\frac{1}{2}<P \bar{e}, \dot{\bar{e}}> \\
& =-\frac{1}{2}<Q \bar{e}, \bar{e}>+<b_{c}^{T} P \bar{e}, g\left(u^{*}-u_{c}\right)> \\
& -<b_{c}^{T} P \bar{e}, g u_{s}>.
\end{aligned}
$$

If the following constraints are known, $0<g_{l} \leq g(x) \leq g_{u}$ (in our approach $0<g_{l} \leq|g(x)| \leq g_{u}$ ) and $|f(x)|<f_{u}$, then according to 11 ,

$$
\left|u^{*}\right| \leq g_{l}^{-1}\left(\left|y_{r e f}^{(n)}\right|+f_{u}+|<\bar{k}, \bar{e}>|\right)
$$

hence, an upper estimation can be given to the derivative of the (19) Lyapunov function in order to investigate the condition $\frac{\mathrm{d} V}{\mathrm{~d} t}<0$, which guarantees closed-loop stability,

$$
\begin{aligned}
\frac{\mathrm{d} V}{\mathrm{~d} t} & \leq-\frac{1}{2}<Q \bar{e}, \bar{e}> \\
& +\left|b_{c}^{T} P \bar{e}\right| g\left\{\left|u_{c}\right|+g_{l}^{-1}\left(\left|y_{r e f}^{(n)}\right|+f_{u}+|<\bar{k}, \bar{e}>|\right)\right\} \\
& -<b_{c}^{T} P \bar{e}, g u_{s}>
\end{aligned}
$$

and since $g g_{l}^{-1}>1$, the supervisory control can be determined accordingly [25]

$$
u_{s}:=\operatorname{sgn}\left(b_{c} P \bar{e}\right)\left\{\left|u_{c}\right|+g_{l}^{-1}\left(\left|y_{r e f}^{(n)}\right|+f_{u}+|<\bar{k}, \bar{e}>|\right)\right\} .
$$

The supervisory control is active only if $V>V_{0}$; the threshold $V_{0}$ has to be customized to the problem. If the expression of the supervisory control is substituted into (21), the result is

$$
\frac{\mathrm{d} V}{\mathrm{~d} t} \leq-\frac{1}{2}<Q \bar{e}, \bar{e}>
$$

thus the condition of Lyapunov stability is fulfilled, the stability of the closed-loop system is guaranteed.

\subsection{Adaptation law}

The nominal control $u_{c}$, based on a zero-order Sugenosystem [27], can be calculated according to the fuzzy relations,

$$
\begin{aligned}
u_{c} & =\varphi^{T}(x) \vartheta \\
{[\varphi(x)]_{k} } & =\frac{\prod_{i=1}^{n} \exp \left(-\left(\frac{x_{i}-\bar{x}_{i}^{k}}{\sigma_{i}^{k}}\right)^{2}\right)}{\sum_{l=1}^{M} \prod_{i=1}^{n} \exp \left(-\left(\frac{x_{i}-\bar{x}_{i}^{l}}{\sigma_{i}^{l}}\right)^{2}\right)}
\end{aligned}
$$

where $[\varphi(x)]_{k}$ weights the $\vartheta^{k}$ output membership function parameter with the distance between the current $x_{i}$ and the expected value of the input membership functions $\bar{x}_{i}^{k}$. In the observed case, $n=2$ and $x_{1}=e$ is the tracking error, $x_{2}=\dot{e}$ is the derivative of the error; $M$ stands for the number of relations which equals to the number of output parameters to be tuned. The best approximation of the ideal control input is

$$
\begin{aligned}
& u_{c}^{*}=\varphi^{T}(x) \vartheta^{*} \\
& \vartheta^{*}:=\arg \min _{\vartheta} \sup _{x}\left|\varphi^{T}(x) \vartheta-u^{*}(x)\right| .
\end{aligned}
$$

To create the adaptation law, the Lyapunov equation has to be improved considering the error of the $\vartheta$ parameter tuning, which can be penalized with the $\gamma$ coefficient as follows. Let

$$
w=u^{*}-u_{c}^{*}
$$

then

$$
\begin{aligned}
u^{*}-u_{c} & =u^{*}-u_{c}^{*}+u_{c}^{*}-u_{c} \\
& =w-\varphi^{T}(x)\left(\vartheta^{*}-\vartheta\right) \\
& =w-\varphi^{T}(x) \Delta \vartheta
\end{aligned}
$$

and the differential equation of the tracking error becomes

$$
\dot{\bar{e}}=\Lambda_{c} \bar{e}+b_{c} g\left\{w-\varphi^{T}(x) \Delta \vartheta-u_{s}\right\}
$$

Thus, the Lyapunov function is

$$
V=\frac{1}{2}<P \bar{e}, \bar{e}>+\frac{1}{2 \gamma}\langle\Delta \vartheta, \Delta \vartheta\rangle
$$

whose derivative has to be examined to investigate the effects of parameter tuning on the stability of the closed-loop system. The derivative of the Lyapunov function is

$$
\frac{\mathrm{d} V}{\mathrm{~d} t}=\frac{1}{2}<P \dot{\bar{e}}, \bar{e}>+\frac{1}{2}<P \bar{e}, \dot{\bar{e}}>+\frac{1}{\gamma}<\Delta \dot{\vartheta}, \Delta \vartheta>
$$

Substituting (30), the expression becomes

$$
\begin{aligned}
\frac{\mathrm{d} V}{\mathrm{~d} t} & =\frac{1}{2} \underbrace{<\left(\Lambda_{c}^{T} P+P \Lambda_{c}\right) \bar{e}, \bar{e}>}_{<0}+\underbrace{<b_{c}^{T} P \bar{e}, g w>}_{=O(w)} \\
& \underbrace{-<b_{c} P \bar{e}, g u_{s}>}_{<0}+\frac{1}{\gamma}<\Delta \dot{\vartheta}-\gamma \varphi g b_{c}^{T} P \bar{e}, \Delta \vartheta>
\end{aligned}
$$

where the last term can be turned to zero to secure closed-loop stability with the appropriate adaptation law as

$$
\Delta \dot{\vartheta}=\dot{\vartheta}:=\gamma \varphi g b_{c}^{T} P \bar{e}
$$

Since $g(x)$ is unknown, $0<g_{l} \leq|g(x)|=g_{0}$ constant approximation is needed, thus the adaptation law becomes

$$
\dot{\vartheta}=\gamma g_{0} \varphi b_{c}^{T} P \bar{e}=\tilde{\gamma} \varphi b_{c}^{T} P \bar{e},
$$

where $\tilde{\gamma}$ is the chosen step size of the adaptation.

The architecture of the closed-loop system and the structure of the adaptive fuzzy controller is detailed in Fig.2 


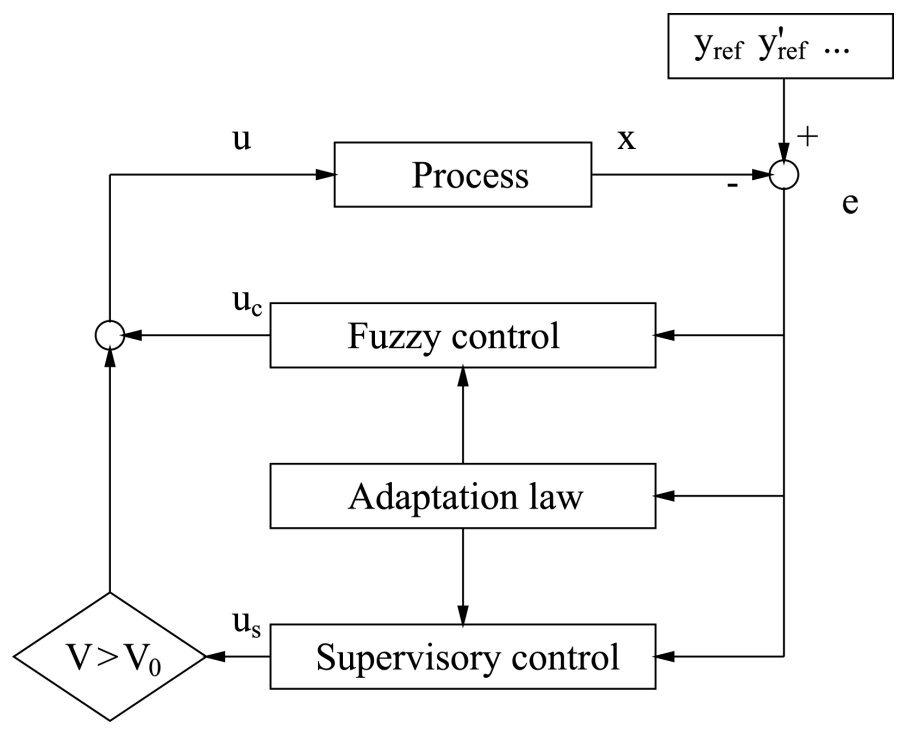

Fig. 2. The interconnection of the closed-loop system

\subsection{Simulation Parameters}

The error dynamics of the closed-loop system is prescribed based on the results of operating point linearization [9], the poles of the linearized model are 6.2526 and -0.3436 . Error dynamics and the time constant of the reference signal (9) are set faster than the stable pole of the system at the initial working point, and the $Q$ matrix is set faster accordingly, since the eigenvalues of the $Q$ matrix determine the stability related system transients.

$$
\begin{aligned}
k_{1} & =1.2 & k_{2} & =0.35 \\
s_{1} & =-0.5 & s_{2} & =-0.7 \\
Q & =\left[\begin{array}{cc}
0.8 & 0 \\
0 & 1
\end{array}\right] & T & =1 / 0.35
\end{aligned}
$$

The input variables are the tracking error and its derivative. The parameters (expected value and variation) of the Gaussian input membership functions and the initial values of the output membership functions to be tuned are presented in Fig. 3 The controller is active only if the tracking error is non-positive, since positive tracking error means that the tumor volume decreased under the prescribed reference value during treatment, which is advantageous in the observed case. The input membership functions of the tracking error were developed according to two aspects: we expect that tracking error varies in the $\left[\begin{array}{ll}-50 & 0\end{array}\right] \mathrm{mm}^{3}$ domain, thus membership functions are set up more dense in this domain to achieve a sensitive controller; on the other hand, the controller must be prepared to handle larger deteriorations caused by parametric changes, which is secured by the membership functions in the $\left[\begin{array}{ll}-800 & -50\end{array}\right] \mathrm{mm}^{3}$ domain. In the case of the derivative related membership functions, the Gaussian which fires during interference shows only the direction of the tracking error change (increase, decrease, no change).

The initial values of the consequent parts of the relations (the output membeship functions) are set based on a priori information, these are the additive inverses of the reasonable serum lev-

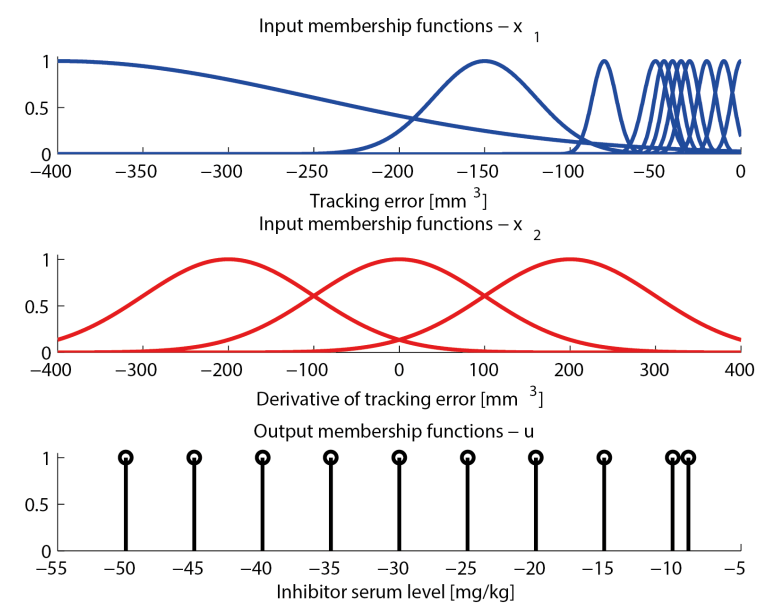

Fig. 3. Input membership functions and the initial values of the output membership functions

els $(50 \mathrm{mg} / \mathrm{kg}$ is the upper limit and $8.85 \mathrm{mg} / \mathrm{kg}$ is the lower limit according to [10]), the negative values are needed because the control design is carried out to determine $-u$ according to (8).

The initial fuzzy rules, whose consequence parts will be adaptated, are presented in Table 1. E refers to the tracking error related membership functions, and DE refers to the derivative related membership functions. The characteristics of the $\mathrm{E} / \mathrm{DE}$ Gaussian are presented in the form: $\mathrm{E} / \mathrm{DE}[\mu \sigma]$, where $\mu$ is the expected value, and $\sigma$ stands for the variation. The interpretation of Table 1 is: if $e$ is near the E Gaussian in the $i^{\text {th }}$ row, and $\dot{e}$ is near the DE Gaussian in the $j^{t h}$ column, then the output $u$ is the $(i, j)$ cell of the table. Rules can be represented through an example as follows: if the tracking error is near the domain of the membership function $\mathrm{E}[-455.5]$ and its derivative is near the domain of the membership function DE[-200 100], then the output serum level is $-40 \mathrm{mg} / \mathrm{kg}$. Each rule which fires during the interference, influences the value of the controller output and will be adaptated. In general, rules express that

- if the tracking error is large, then $u$ is as high as possible

- if the tracking error is in the medium or low range and increases, then $u$ is higher

- if the tracking error is in the medium or low range and does not change, then $u$ does not change (the serum level is maintained which is able to compensate the error)

- if the tracking error is in the medium or low range and decreases, then $u$ is lower.

We can summarize these rules such that if the tracking error is not large, and changes in the right direction (decreases), then the controller output can be decreased, otherwise the controller output has to be increased or at least left unchanged.

The adaptation law and the supervisory control related parameters were set according to results of exact linearization [12]. 
Tab. 1. The initial fuzzy rules to be adaptated

\begin{tabular}{|c|c|c|c|}
\hline & $\mathrm{DE}\left[\begin{array}{lll}200 & 100\end{array}\right]$ & $\mathrm{DE}\left[\begin{array}{ll}0 & 100\end{array}\right]$ & DE $\left[\begin{array}{lll}-200 & 100]\end{array}\right.$ \\
\hline$E\left[\begin{array}{lll}-400 & 150]\end{array}\right.$ & -50 & -50 & -50 \\
\hline$E\left[\begin{array}{lll}-150 & 30\end{array}\right]$ & -50 & -50 & -50 \\
\hline$E\left[\begin{array}{lll}-80 & 7\end{array}\right]$ & -50 & -50 & -50 \\
\hline$E\left[\begin{array}{lll}-50 & 7\end{array}\right]$ & -50 & -50 & -45 \\
\hline$E\left[\begin{array}{lll}-45 & 5.5]\end{array}\right.$ & -45 & -45 & -40 \\
\hline$E\left[\begin{array}{lll}-40 & 5.5]\end{array}\right.$ & -35 & -30 & -25 \\
\hline $\mathrm{E}\left[\begin{array}{lll}-35 & 5.5] \\
\end{array}\right.$ & -30 & -25 & -20 \\
\hline$E\left[\begin{array}{lll}-30 & 5.5]\end{array}\right.$ & -25 & -25 & -20 \\
\hline$E\left[\begin{array}{lll}-20 & 5.5]\end{array}\right.$ & -20 & -20 & -15 \\
\hline $\mathrm{E}[-105.5]$ & -15 & -10 & -10 \\
\hline$E\left[\begin{array}{ll}0 & 5.5\end{array}\right]$ & -10 & -8.85 & -8.85 \\
\hline
\end{tabular}

The real-valued functions of the system transformed into the form of (7) are

$$
\begin{aligned}
& f(x)=\left(\lambda \ln \frac{x_{1}}{x_{2}}+\lambda\right) \lambda x_{1} \ln \frac{x_{1}}{x_{2}}+\lambda x_{1} \frac{1}{x_{2}}\left(\tilde{b} x_{1}-\tilde{d} x_{1}^{\frac{2}{3}} x_{2}\right) \\
& g(x)=-\tilde{e} \lambda x_{1},
\end{aligned}
$$

where the value of $|g|$ varies in [0.1267 253], the average value of $|g|$ was set accordingly, $f_{u}$ was found to be $5 \cdot 10^{6}$, which influences the magnitude of the controller output of the supervisory control. The threshold of the supervisory control activization is set as high as possible, since if it is active, the controller output is increased with orders of magnitude which is opposed to the physiological constraints. The numerical values of these parameters are chosen as follows:

$$
\begin{aligned}
& g_{0}=50 \quad g_{l}=0.1267 \quad g_{u}=253 \\
& \gamma=5 \cdot 10^{-4} f_{u}=5 \cdot 10^{6} \quad V_{0}=10^{6} \text {. }
\end{aligned}
$$

\section{Simulation results}

During the simulations, the observed tumor domain is in the range of $0-2000 \mathrm{~mm}^{3}$. Since antiangiogenic therapy is only able to reduce the vascular endothelium grown by the tumor itself, the possible minimal tumor volume is set to $1 \mathrm{~mm}^{3}$. The tumor reaches this minimal volume in an avascular state which can be maintained with a relatively low constant serum level $(8.85 \mathrm{mg} / \mathrm{kg})$, the exact value of the mentioned serum level can be derived from the steady-state equations of the model as in [10].

We expect that tumor volume decreases to the minimal volume as fast as possible such that the applied serum level does not exceed $50-55 \mathrm{mg} / \mathrm{kg}$ while keeping the total inhibitor inlet as low as possible.

Simulation results are shown in Fig. 4 and Fig. 5 represents how output parameters change in course of the adaptation. Tumor regresses to $1 \%$ of initial tumor volume after 21 days, fast regression is achieved and the control input exceeds $40 \mathrm{mg} / \mathrm{kg}$ only for 7 days. After the first 40 days tumor reaches $5 \mathrm{~mm}^{3}$, and the minimal volume reached in 50 days is $3.5 \mathrm{~mm}^{3}$. The total inhibitor inlet is $996.8 \mathrm{mg} / \mathrm{kg}$. The control input varies between $9 \mathrm{mg} / \mathrm{kg}$ and $50 \mathrm{mg} / \mathrm{kg}$, and no saturation was needed to
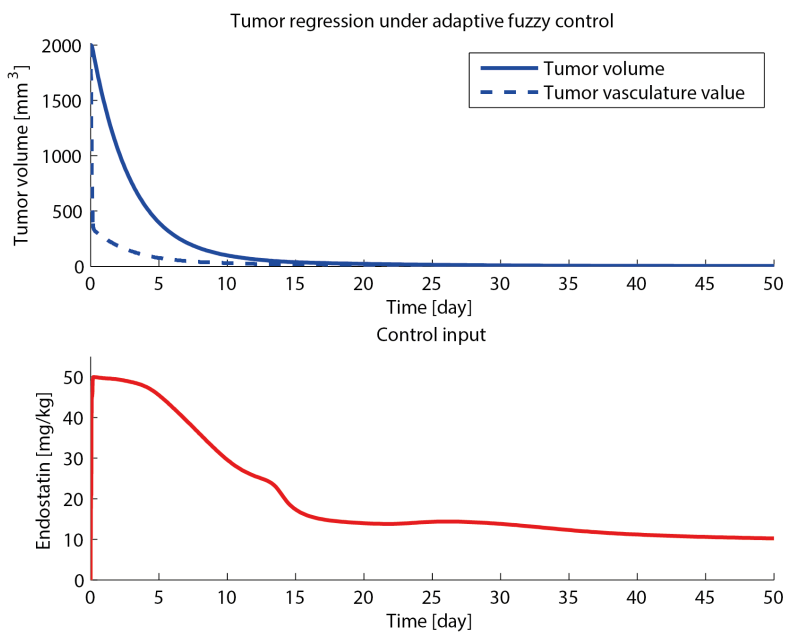

Fig. 4. Tumor regression and control input under adaptive fuzzy control

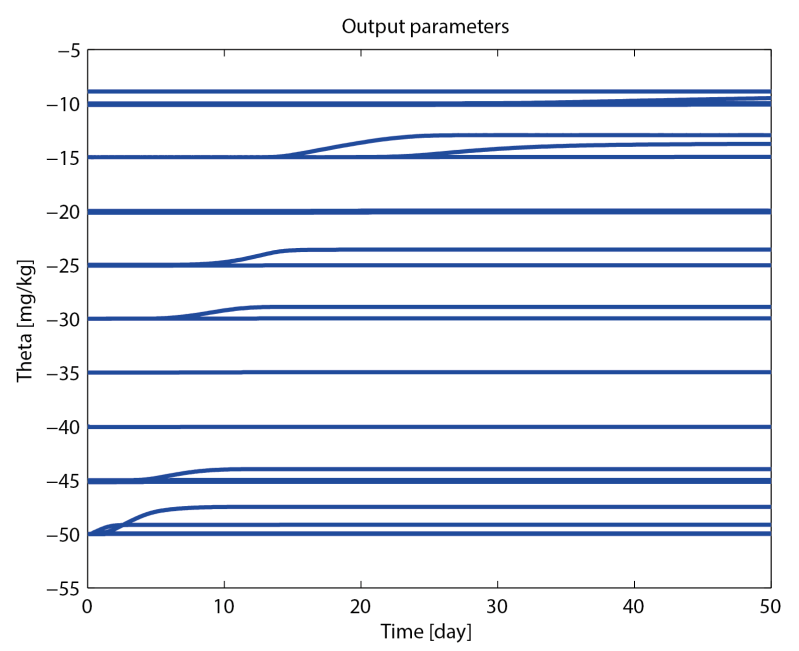

Fig. 5. Adaptation of output parameters

fulfill the physiological constraints opposed to the control inputs in [7,9].

In terms of daily and total inlet, adaptive fuzzy control outperformed the controllers presented in [6, 8], and the plateau reached is far lower as well. The therapy presented in this paper causes less strain on the body since control input exceeds $40 \mathrm{mg} / \mathrm{kg}$ only for 7 days, not for 22 days as in [9]. Total inihibitor inlet is not significantly lower than in [7,9,10], however the need of saturation could be totally eliminated. The minimal volume plateau was reached, the controller performed better in this aspect as well than the controllers presented in [7, 9, 10].

\subsection{The effect of parameter perturbation}

Model related parameters $(\lambda, \tilde{b}, \tilde{d}, \tilde{e})$ were perturbed independently with a variability of $\pm 25 \%$ to investigate the effects of model parametric changes. Simulations show that the performance of tumor regression does not change significantly, which is a prosperous result. Observing Fig. 6 and Fig. 7 one can see that in some cases the effects of model parametric changes turn 

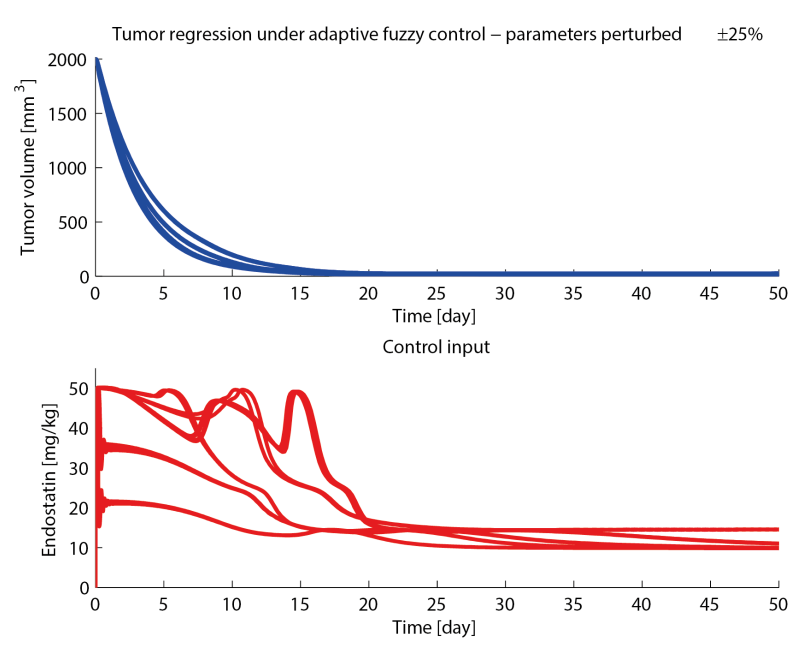

Fig. 6. Tumor regression and control inputs in the case of model parameter perturbation with a variability of $\pm 25 \%$

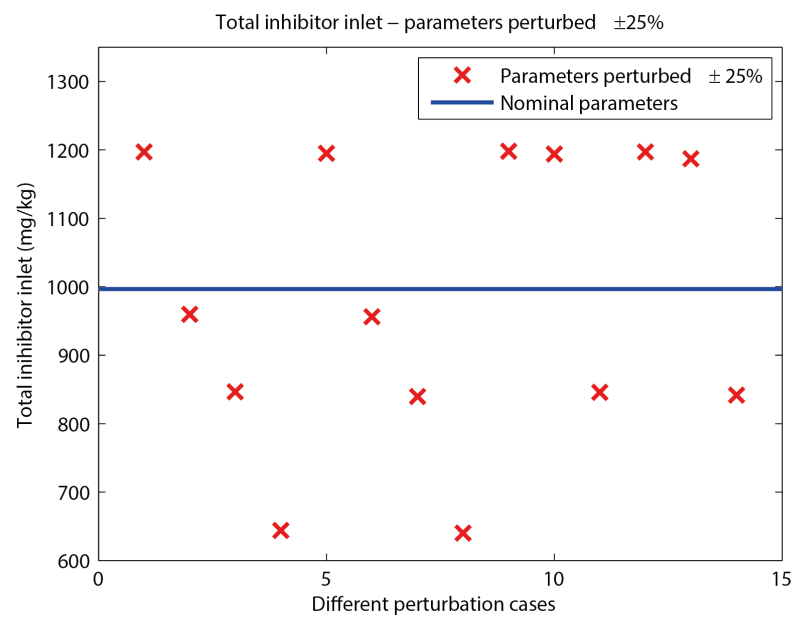

Fig. 7. Total inhibitor inlet in the case of model parameter perturbation with a variability of $\pm 25 \%$

to be advantageous, since both daily and total inlet decreased compared to the original case. The latter phenomenon, that the controller does not exceed the optimal serum level, can be explained with the efficiency of the adaptation. The inhibitor inlets of the disadvantegous cases are still far lower than the inlets in [6, 8] and close to the inlets of the controllers presented in [7],9], and physiological constraints were satisfied without the necessity of external saturation. Thus, better performance was achieved even in the perturbed cases.

\section{Conclusion}

In this paper, adaptive fuzzy control design was carried out to attain tumor regression using low inhibitor serum levels.

Fast tumor regression and low daily and total inhibitor inlets were achieved. As a benefit of the adaptation, the performance of tumor regression does not significantly change because of parameter perturbations even with a relatively high variability. The designed controller outperformed control strategies published by the authors [7, 9, 10] and other researchers [6, 8] in the aspects investigated in this paper.

However, the controller was not able to reach the minimal volume $\left(1 \mathrm{~mm}^{3}\right)$ in 50 days as controllers in [11, 12]; the plateau can be attained after 100 days.

The effects of model parameter perturbations were investigated, since the advantage of adaptive fuzzy methodology is that controller design can be carried out without knowing the exact model formulation using appropriate estimation. On the other hand, if the nominal model parameters change during the control process, the controller is able to adaptate to the changes.

The aim of further research is to pose a new biologically validated mathematical model which involves more complex characteristics of tumor growth dynamics.

\section{Acknowledgement}

L. Kovács is supported by the János Bolyai Research Scholarship of the Hungarian Academy of Sciences. A. Szeles is supported by the European Union and the State of Hungary, co-financed by the European Social Fund in the framework of TÁMOP 4.2.4. A/1-11-1-2012-0001 „National Excellence Program“.

\section{References}

1 Wu H C, Huang C T, Chang D K, Anti-Angiogenic Therapeutic Drugs for Treatment of Human Cancer, Journal of Cancer Molecules, 4(2), (2008), 3745.

2 Gotink K J, Verheul H M W, Anti-angiogenic tyrosine kinase inhibitors: what is their mechanism of action?, Angiogenesis, 13(1), (2010), 1-14, DOI 10.1007/s10456-009-9160-6

3 Hahnfeldt P, Panigrahy D, Folkman J, Hlatky L, Tumor Development under Angiogenic Signaling: A Dynamical Theory of Tumor Growth, Treatment Response, and Postvascular Dormancy, Cancer Research, 59(19), (1999), 4770-4775.

4 d'Onofrio A, Cerrai P, A bi-parametrical model for the tumour angiogenesis and antiangiogenesis therapy, Mathematical and Computer Modelling, 49(5-6), (2009), 1156-1163, DOI 10.1016/j.mcm.2008.05.001

5 d'Onofrio A, Gandolfi A, Rocca A, The dynamics of tumourvasculature interaction suggests low-dose, time-dense anti-angiogenic schedulings, Cell Proliferation, 42(3), (2009), 317-329, DOI $10.1111 / \mathrm{j} .1365-$ 2184.2009.00595.x

6 Ledzewicz U, Schättler H, A Synthesis of Optimal Controls for a Model of Tumor Growth under Angiogenic Inhibitors, In: Proceedings of the 44th IEEE Conference on Decision and Control, and the European Control Conference, 2005, pp. 934-939, DOI 10.1109/CDC.2005.1582277

7 Drexler D A, Kovács L, Sápi J, Harmati I, Benyó Z, Model-based analysis and synthesis of tumor growth under angiogenic inhibition: a case study, In: Proceedings of the 18th World Congress of the International Federation of Automatic Control; Milano, Italy, 2011, pp. 3753-3758.

8 Kassara K, Moustafid A, Angiogenesis inhibition and tumor-immune interactions with chemotherapy by a control set-valued method, Mathematical Biosciences, 231(2), (2011), 135-143, DOI 10.1016/j.mbs.2011.02.010

9 Szeles A, Sápi J, Drexler D A, Harmati I, Sápi Z, Kovács L, Modelbased Angiogenic Inhibition of Tumor Growth using Modern Robust Control Method, In: Proceedings of the 8th IFAC Symposium on Biological and Medical Systems; Budapest, Hungary, 2012, pp. 113-118. 
10 Sápi J, Drexler D A, Harmati I, Sápi Z, Kovács L, Linear state-feedback control synthesis of tumor growth control in antiangiogenic therapy, In: Proceedings of the 10th IEEE Jubilee International Symposium on Applied Machine Intelligence and Informatics; Herl'any, Slovakia, January 26-28, 2012, pp. 143-148.

11 Drexler D A, Sápi J, Szeles A, Harmati I, Kovács A, Kovács L, Flat control of tumor growth with angiogenic inhibition, In: Proceedings of the 7th International Symposium on Applied Computational Intelligence and Informatics; Timisora, Romania, 2012, pp. 179-183.

12 Szeles A, Drexler D A, Sápi J, Harmati I, Kovács L, Model-based Angiogenic Inhibition of Tumor Growth using Feedback Linearization, In: Proceedings of the 52nd IEEE Conference on Decision and Control; Florence, Italy, December 10-13, 2013, pp. 2054-2059.

13 Holland J F, Frei E, Cancer Medicine, 6th edition, BC Decker Inc.; Hamilton, Ontario, 2003.

14 Connell P P, Hellman S, Advances in Radiotherapy and Implications for the Next Century: A Historical Perspective, Cancer Research, 69(2), (2009), 383-392, DOI 10.1158/0008-5472.CAN-07-6871

15 Perry M C, The Chemotherapy Source Book, 4th, Lippincott Williams and Wilkins, 2008.

16 Pollock R E, Advanced Therapy in Surgical Oncology, BC Decker, Hamilton, Ontario, Canada, 2008.

17 Lippert T H, Ruoff H J, Volm M, Current Status of Methods to Assess Cancer Drug Resistance, International Journal of Medical Sciences, 8(3), (2011), 245-253, DOI $10.7150 /$ ijms.8.245

18 Pluda J M, Tumor-associated angiogenesis: mechanisms, clinical implications, and therapeutic strategies, Seminars in Oncology, 24(2), (1997), 203218.

19 Kerbel R, A cancer therapy resistant to resistance, Nature, 390(6658), (1997), 335-336, DOI $10.1038 / 36978$

20 Qian Y Y, Zhang H, Hou Y, Yuan L, Li G Q, Guo S Y, Tadashi H, Liu $\mathbf{Y} \mathbf{Q}$, Celastrus Orbiculatus extract inhibits tumor angiogenesis by targeting vascular endothelial growth factor signaling pathway and shows potent antitumor activity in hepatocarcinomas in Vitro and in Vivo, Chinese Journal of Integrative Medicine, 18(10), (2012), 752-760, DOI 10.1007/s11655-0110819-7

21 Loges S, Schmidt T, Carmeliet P, Mechanisms of Resistance to Anti-Angiogenic Therapy and Development of Third-Generation AntiAngiogenic Drug Candidates, Genes \& Cancer, 1(1), (2010), 12-25, DOI 10.1177/1947601909356574

22 O'Reilly M S, Boehm T, Shing Y, Fukai N, Vasios G, Lane W S, Flynn E, Birkhead J R, Olsen B R, Folkman J, Endostatin: An Endogenous Inhibitor of Angiogenesis and Tumor Growth, Cell, 88(2), (1997), 277-285, DOI 10.1016/S0092-8674(00)81848-6

23 Ellis L M, Haller D G, Bevacizumab Beyond Progression: Does This Make Sense?, Journal of Clinical Oncology, 26(33), (2008), 5313-5315, DOI 10.1200/JCO.2008.17.4540

24 Zadeh L A, Fuzzy sets, Information and Control, 8(3), (1965), 338-353, DOI 10.1016/S0019-9958(65)90241-X

25 Lantos B, Fuzzy Systems and Genetic Algorithms, Múegyetemi Kiadó; Budapest, 2002.

26 Wang L X, Adaptive fuzzy systems and control, Prentice Hall, 1994.

27 Takagi T, Sugeno M, Fuzzy identification of systems and its applications to modeling and control, IEEE Transactions on Systems, Man, and Cybernetics, 15(1), (1985), 116-132, DOI 10.1109/TSMC.1985.6313399 